\title{
Jatropha curcas ortholog of tomato MADS-box gene 6 (JCTM6) promoter exhibits floral-specific activity in Arabidopsis thaliana
}

\author{
Jing-Xian Wang ${ }^{1,2}$, Xin Ming ${ }^{1,2}$, Yan-Bin Tao ${ }^{\text {Corresp., } 2,3}$, Zeng-Fu Xu ${ }^{\text {Corresp. } 2,3}$ \\ ${ }^{1}$ School of Life Sciences, University of Science and Technology of China, Hefei, Anhui, China \\ 2 CAS Key Laboratory of Tropical Plant Resources and Sustainable Use, Xishuangbanna Tropical Botanical Garden, Innovation Academy for Seed \\ Design,Chinese Academy of Sciences, Xishuangbanna, Menglun, Mengla, Yunnan, China \\ 3 \\ Center of Economic Botany, Core Botanical Gardens, Chinese Academy of Sciences, Menglun, Mengla, Yunnan, China \\ Corresponding Authors: Yan-Bin Tao, Zeng-Fu Xu \\ Email address: taoyanbin@xtbg.ac.cn, zfxu@xtbg.ac.cn
}

Background. Jatropha curcas L., a perennial oilseed plant, is considered as a promising feedstock for biodiesel production. Genetic modification of flowering characteristics is critical for Jatropha breeding. However, analysis floral-specific promoters in Jatropha is limited.

Methods. In this study, we isolated the Jatropha ortholog of TM6 (JCTM6) gene from Jatropha flower CDNA library and detect the expression pattern of JCTM6 gene by quantitative reverse transcriptionpolymerase chain reaction (qRT-PCR). We isolated a 1.8-kb fragment from the 5 ' region of the JcTM6 gene and evaluated its spatiotemporal expression pattern in Arabidopsis using the $\beta$-glucuronidase (GUS) reporter gene and Arabidopsis ATP/ADP isopentenyltransferase 4 (AtIPT4) gene, respectively.

Results. JCTM6 was identified as a flower-specific gene in Jatropha. As expected, JcTM6 promoter was only active in transgenic Arabidopsis flowers with the strongest activity in stamens. Moreover, JCTM6:AtIPT4 transgenic Arabidopsis showed a phenotype of large flowers without any alterations in other organs. Furthermore, deletion of the region from $-1,717$ to $-876 \mathrm{bp}$ resulted in the disappearance of promoter activity in stamens but an increase in promoter activity in young leaves, sepals, and petals. Deletion analysis suggests that the -1,717- to -876-bp promoter fragment contains regulatory elements that confer promoter activity in stamens and inhibit activity in young leaves, sepals, and petals. 
1 Jatropha curcas TOMATO MADS-BOX GENE 6

2 (JcTM6) promoter exhibits floral-specific activity in

3 Arabidopsis thaliana

4

5

6

7

8

12

Jing-Xian Wang ${ }^{1,2}$, Xin Ming ${ }^{1,2}$, Yan-Bin Tao ${ }^{2,3, *}$, and Zeng-Fu Xu ${ }^{2,3, *}$

${ }^{1}$ School of Life Sciences, University of Science and Technology of China, Hefei, Anhui, China

${ }^{2}$ CAS Key Laboratory of Tropical Plant Resources and Sustainable Use, Xishuangbanna Tropical

Botanical Garden, Innovation Academy for Seed Design, Chinese Academy of Sciences,

Menglun, Mengla, Yunnan, China

${ }^{3}$ Center of Economic Botany, Core Botanical Gardens, Chinese Academy of Sciences, Menglun, Mengla, Yunnan, China

Corresponding authors:

Yan-Bin Tao

88 Xuefu road, Kunming, Yunnan, 666303, China

Email address: taoyanbin@xtbg.ac.cn

Zeng-Fu Xu

88 Xuefu road, Kunming, Yunnan, 666303, China

Email address: zfxu@xtbg.ac.cn

\section{Abstract}

Background. Jatropha curcas L., a perennial oilseed plant, is considered as a promising feedstock for biodiesel production. Genetic modification of flowering characteristics is critical for Jatropha breeding. However, analysis floral-specific promoters in Jatropha is limited. Methods. In this study, we isolated the Jatropha ortholog of TM6 (JcTM6) from Jatropha flower cDNA library and detect the expression pattern of JcTM6 gene by quantitative reverse transcription-polymerase chain reaction (qRT-PCR). We isolated a 1.8-kb fragment from the $5^{\prime}$ region of the JCTM6 gene and evaluated its spatiotemporal expression pattern in Arabidopsis using the $\beta$-glucuronidase (GUS) reporter gene and Arabidopsis ATP/ADP isopentenyltransferase 4 (AtIPT4) gene, respectively.

Results. JcTM6 was identified as a flower-specific gene in Jatropha. As expected, JcTM6 promoter was only active in transgenic Arabidopsis flowers with the strongest activity in stamens. Moreover, JcTM6:AtIPT4 transgenic Arabidopsis showed a phenotype of large flowers without any alterations in other organs. Furthermore, deletion of the region from $-1,717$ to -876 bp resulted in the disappearance of promoter activity in stamens but an increase in promoter activity in young leaves, sepals, and petals. Deletion analysis suggests that the $-1,717$ to $-876-b p$ promoter fragment contains regulatory elements that confer promoter activity in stamens and inhibit activity in young leaves, sepals, and petals. 


\section{Introduction}

41 Promoter plays a significant role in gene expression regulation. Three types of promoters are

42

43

44

45

46

47

48

49

50

51

52

53

54

55

56

57

58

59

60

61

62

63

64

65

66

67

68

69

70

71

72

73

74

75

76

77

78

79 currently employed in plant genetic engineering, constitutive, tissue-specific, and inducible promoters (Muthusamy et al. 2017; Potenza et al. 2004). Tissue-specific promoters drive transgene expression in a specific spatiotemporal pattern, which is effective in the modification of agronomic traits of crop plants. For example, the rice (Oryza sativa L.) gene OsGA2oxI encodes a gibberellin (GA) catabolic enzyme, GA 2-oxidase (Lester et al. 1999; Martin et al. 1999; Thomas et al. 1999). When the expression of OsGA2oxl was driven by the constitutive Actin promoter, transgenic rice plants failed to set grains. To prevent sterility, the promoter of a GA biosynthesis gene, OsGA3ox2, which encodes GA 3-oxidase and is specifically active in shoots, was used to control the expression of OsGA2oxl. As expected, transgenic rice exhibited a semi-dwarf phenotype with normal yield (Sakamoto et al. 2003). GA 20-oxidase is a GA biosynthetic enzyme in plants (Coles et al. 1999). In poplar (Populus spp.), overexpression of the Pinus densiflora GA 20-oxidase gene (PdGA20ox) under the control of the constitutive $35 S$ promoter increased GA levels, thereby accelerating stem growth and plant biomass; however, transgenic poplar plants showed poor leaf development and root growth. When the PdGA20ox gene was driven by a xylem-specific promoter $D X 15$ from poplar, the undesirable phenotypes were reduced (Jeon et al. 2016).

Physic nut (Jatropha curcas L.) is an oilseed plant belonging to the Euphorbiaceae family. The seed oil of Jatropha is a promising feedstock for biodiesel production (Kumar \& Sharma 2008). However, low seed yield, which is mainly caused by low female: male ratio, is a longstanding problem in Jatropha (Raju \& Ezradanam 2002; Rao et al. 2008). Jatropha is a monoecious plant species with male and female flowers on the same inflorescence, and the average ratio of female to male flowers is 1:13-1:29 (Raju \& Ezradanam 2002; Tewari et al. 2007). There are 100-300 flowers in each inflorescence of Jatropha, which only produce approximately 10 fruits (Kumar \& Sharma 2008; Pan \& Xu 2011). Hence, genetic modification of flowering characteristics is critical for Jatropha breeding. Floral-specific promoters play crucial roles in this modification because they can drive efficient expression of functional genes in flowers without affecting the vegetative growth of plants. In pea (Pisum sativum), the PsEND1 promoter exhibits anther-specific activity. Expression of the ribonuclease gene barnase (Gardner et al. 2009) in Arabidopsis and Brassica napus under the control of the PSEND1 promoter causes anther ablation at an early developmental stage, leading to male sterility (Roque et al. 2007). Arabidopsis APETALA3 (AP3) promoter was identified as a floral-specific promoter in petunia (Petunia $x$ hybrida). Expression of the Agrobacterium tumefaciens isopentenyltransferase (ipt) gene under the control of the AtAP3 promoter in petunia increased the flower size, without affecting vegetative development (Verdonk et al. 2008). However, analysis of promoters, especially floral-specific promoters, in Jatropha is limited. Although the Jatropha APETALA1 $(J c A P 1)$ promoter was recently identified as a reproductive tissue-specific promoter showing high activity in inflorescence buds and seeds (Tao et al. 2016), it is not sufficient to address transgene expression analysis in Jatropha. 
80

81

82

83

84

85

86

87

88

89

90

91

92

93

94

95

96

97

98

99

100

101

102

103

104

105

106

107

108

109

110

111

112

113

114

115

116

117

118

119

In this study, we isolated the promoter of the Jatropha ortholog of TOMATO MADS-BOX GENE 6 (JCTM6), a floral-specific gene. The activity of JcTM6 promoter was evaluated in Arabidopsis using the $\beta$-glucuronidase (GUS) reporter gene. The results of GUS staining showed that the JcTM6 promoter was active only in flowers, with the highest activity in stamens. By using this promoter directed a cytokinin biosynthesis gene, Arabidopsis ATP/ADP isopentenyltransferase 4 (AtIPT4) gene ( $\mathrm{Li}$ et al. 2010), only flower phenotype was changed in transgenic Arabidopsis. Furthermore, deletion analysis showed that an approximately $0.85-\mathrm{kb}$ fragment of the JcTM6 promoter ( -1717 to $-876 \mathrm{bp}$ ) is critical for maintaining its floral-specific expression pattern.

\section{Materials \& Methods}

\section{Plant materials}

Plants of Jatropha curcas and Arabidopsis thaliana ecotype Columbia (Col-0) were used in this study. Jatropha plants were cultivated in Xishuangbanna, Yunnan Province, China, as described previously (Pan \& Xu 2011). Arabidopsis plants were grown in an environmentally controlled room at $22^{\circ} \mathrm{C}$ under $16-\mathrm{h}$ light $/ 8$-h dark photoperiod.

\section{JcTM6 expression analysis}

The JcTM6 gene (GenBank accession no. MN820724) was identified in the Jatropha flower cDNA library (Chen et al. 2014). Quantitative reverse transcription-polymerase chain reaction (qRT-PCR) was performed to examine the expression level of JcTM6 in different organs of Jatropha (roots, stems, young leaves, mature leaves, inflorescence buds, female flowers, male flowers, pericarps and seeds at 42 days after pollination (DAP), male sepals and petals, stamens, female sepals and petals, and pistils) and Arabidopsis (leaves and flowers). Total RNA from each organ was isolated using the silica particle extraction method (Ding et al. 2008). Then, qRT-PCR was performed as previously described in Tao (2015). The JcGAPDH and AtActin were used as an internal control for data normalization. Primers used for qRT-PCR are listed in Table 1. The results of qRT-PCR were obtained from three biological replicates and three technical replicates.

\section{Cloning of the upstream region of JcTM6}

The 5' region of JcTM6 was isolated from Jatropha genomic DNA by genome walking ( et al. 1995) according to the Genome Walker ${ }^{\mathrm{TM}}$ Kit Universal User Manual (Clontech). Then, the full-length JCTM6 promoter was amplified using the primers, XT405 and XT408. The PCR product was cloned into the pGEM-T Easy vector. Putative cis-acting elements in the JcTM6 promoter were analyzed using the PLACE database (Higo et al. 1999). The transcriptional start site of JcTM6was identified as previously described in Tao (2016). Primers employed for genome walking and 5'-RACE are listed in Table 1.

\section{Construction of JcTM6 promoter-GUS fusion and Arabidopsis transformation}


120 To generate the JcTM6:GUS plasmid, $X b a \mathrm{I}$ and BamHI were used to digested pBI101 (Jefferson 121 et al. 1987), and the pGEM -T Easy vector containing the JcTM6 promoter, respectively. The 122 resulting fragments were ligated using the T4 DNA Ligase (Promega) to generate the 123 JcTM6:GUS fusion construct. Then, the JcTM6:GUS plasmid was introduced into 124 Agrobacterium tumefaciens EHA105 by electroporation (GenePulser Xcell; Bio-Rad), and the 125 transformed A. tumefaciens cells were used to transform Arabidopsis plants by the floral dip

126

127

128

129

130

131

132

133

134

135

136

137

138

139

140

141

142

143

144

145

146

147

148

149

150

151

152

153

154

155

156

157

158

159 method (Clough \& Bent 1998).

\section{Histochemical GUS staining assay}

To perform GUS staining, various tissues of transgenic Arabidopsis were submerged in the GUS assay buffer $\left(50 \mathrm{mM}\right.$ sodium phosphate [pH 7.0], $0.5 \mathrm{mM} \mathrm{K}_{3} \mathrm{Fe}(\mathrm{CN})_{6}, 0.5 \mathrm{mM} \mathrm{K}_{4} \mathrm{Fe}$ $(\mathrm{CN})_{6} \cdot 3 \mathrm{H}_{2} \mathrm{O}, 0.5 \%$ Triton $\mathrm{X}-100$, and $1 \mathrm{mM} \mathrm{X}$-Gluc) and vacuum-infiltrated for $15 \mathrm{~min}$. Then, tissues were incubated overnight at $37^{\circ} \mathrm{C}$, cleared in $70 \%$ ethanol (Jefferson et al. 1987), and examined under a stereomicroscope (Leica M80). The results of GUS staining were obtained from five biological replicates and three technical replicates.

\section{Results}

\section{JcTM6 expression in Jatropha}

We identified the JcTM6 cDNA (GenBank accession no. MN820724) from our Jatropha flower cDNA library constructed previously (Chen et al. 2014). JcTM6 encodes a 230-amino acid protein, which shows high similarity to TM6 homologs from other plant species (Fig. 1A). Phylogenetic analyses showed that JcTM6, which contains the paleoAP3 motif, belongs to the TM6 group, rather than the euAP3 group (Fig. 1B).

To analyze the expression pattern of JcTM6 in Jatropha, qRT-PCR was performed using total RNA extracted from various tissues including roots, stems, leaves, inflorescences, female and male flowers, and pericarps and seeds at 42 DAP. The JcTM6 gene was predominantly expressed in female and male flowers (Fig. 2), indicating that JcTM6 is a flower-specific gene. Furthermore, JcTM6 showed high expression in the stamens of male flowers and petals of male and female flowers but low expression in sepals and pistils (Fig. 2). Thus, the expression pattern of JcTM6 in floral organs is consistent with that of class B genes (Weigel \& Meyerowitz 1994).

\section{Isolation and sequence analysis of JcTM6 promoter}

A 1.8-kb fragment of the JcTM6 promoter (Fig. 3A, -1717 to +103 bp; GenBank accession no. MN044579) was isolated from Jatropha genomic DNA by genome walking (Siebert et al. 1995). The transcription start site of JcTM6 was located $103 \mathrm{nt}$ upstream of the translation start codon (Fig. 3A). Analysis of the JcTM6 promoter using the PLACE database (Higo et al. 1999) revealed various putative cis-elements in the 1.8-kb JcTM6 promoter fragment (Fig. 3A) including two CArG boxes, which act as binding sites for MADS-box transcription factors (Irish \& Yamamoto 1995), some pollen-specific elements, including five GTGANTG10 motifs (GTGA) and eight POLLEN1LELAT52 motifs (AGAAA) (Muschietti et al. 1994; Rogers et al. 
160

161

162

163

164

165

166

167

168

169

170

171

172

173

174

175

176

177

178

179

180

181

182

183

184

185

186

187

188

189

190

191

192

193

194

195

196

197

198

199

2001), and a Q element (TGACCT), which shows enhancer-like activity for the pollen-specific expression of maize (Zea mays L.) ZM13 gene (ㅂamilton et al. 1998).

\section{Activity of the JcTM6 promoter in Arabidopsis}

To detect the activity of JcTM6 promoter, a JcTM6 promoter-GUS fusion construct (Fig. 3B) was expressed in Arabidopsis, and GUS staining was monitored in homozygous T3 plants (Fig. 4). No GUS staining was observed in 10-day-old Arabidopsis seedlings (Fig. 4A). Among the five tissues of adult plants examined (including roots, stems, leaves, flowers, and green siliques) GUS staining was detected only in flowers (Fig. 4B-G). Among all floral organs, GUS staining intensity was the strongest in stamens, followed by sepals and petals, with faint staining in carpels (Fig. S1). Based on the results of GUS staining, we conclude that the JcTM6 promoter functions as a flower-specific promoter in Arabidopsis.

\section{Deletion analysis of the JcTM6 promoter}

To analyze the region essential for flower-specific activity of the JcTM6 promoter, we carried out a deletion analysis. A deletion variant of the JcTM6 promoter lacking the region from $-1,717$ to $-876 \mathrm{bp}$ was fused to the GUS gene and transformed into Arabidopsis (Fig. 5A). Compared with the full-length JcTM6 promoter, the deletion was not only active in flowers but also in young leaves (Fig. 5B). Moreover, the deletion showed no promoter activity in stamens but increased activity in sepals and petals (Fig. 5C and D). These results indicate that the region from $-1,717$ to $-876 \mathrm{bp}$ is critical for JcTM6 promoter activity in stamens and inhibition of promoter activity in young leaves, sepals, and petals.

\section{JcTM6:AtIPT4 transgenic Arabidopsis produced large flowers}

To further verify the floral specificity of JcTM6 promoter, a cytokinin biosynthetic gene (AtIPT4) was expressed under the control of JcTM6 promoter in Arabidopsis. JcTM6:AtIPT4 vector was constructed and was transformed into Arabidopsis plants. A total of 25 independent JcTM6:AtIPT4 lines were obtained. As expected, all transgenic lines showed no vegetative difference from the wild type and most of them produced larger flowers (Fig. 6). Furthermore, the development of siliques was also unaffected. To verify the morphological alteration in flowers that is caused by the transgene, we examined the expression levels of AtIPT4 and the cytokinin signaling genes Arabidopsis histidine kinase 2 (AHK2) (Nishimura et al, 2004) and Arabidopsis response regulator 5 (ARR5) (D'Agostino et al, 2000) in wild type and JcTM6:AtIPT4 transgenic plants. The expression level of AtIPT4 in flowers of transgenic lines is significantly higher than that in wild type, whereas the AtIPT4 expression in the leaves of transgenic plants was not different from that in leaves of wile-type plants (Fig. 7A). As expected, higher expression levels of $A H K 2$ and $A R R 5$ were detected in the flowers of transgenic lines (Fig. 7B). These results indicate that the morphological alteration in flowers of JcTM6:AtIPT4 transgenic plants is caused by the flower-specific expression of the transgene driven by the JCTM6 promoter. JCTM6 promoter is indeed a flower-specific promoter. 


\section{Discussion}

202

203

204

205

206

207

208

209

210

211

212

213

214

215

216

217

218

219

220

221

222

223

224

225

226

227

228

229

230

231

232

233

234

235

236

237

238

239

TM6 is a member of the MADS-box gene family, which belongs to the paleoAP3 lineage (Pnueli et al. 1991; Rijpkema et al. 2006; Wu et al. 2011). In tomato (Solanum lycopersicum) and petunia, TM6 functions as a class B gene that play an essential role in stamen development, although it is mainly expressed in whorls 3 and 4 , similar to a class $C$ gene (de Martino et al. 2006; Rijpkema et al. 2006). In trioecious papaya (Carica papaya) plants, which produce male, female, and hermaphrodite flowers, two TM6 genes were isolated previously (CpTM6-1 and CpTM6-2). Both genes are predominantly expressed in the petals of all sex types and stamens of hermaphrodite and male flowers, although CpTM6-2 is also expressed in leaves (Ackerman et al. 2008). In this study, we identified JcTM6 as a flower-specific gene in Jatropha, with high expression in female and male flowers (Fig. 2). Similar to CpTM6-1, the JcTM6 gene showed high expression in the petals of female and male flowers and stamens of male flowers. Because JCTM6 showed flower-specific expression, we isolated its upstream region from Jatropha genomic DNA and analyzed its activity in Arabidopsis by GUS staining.

In transgenic Arabidopsis, GUS staining showed that the JcTM6 promoter was active only in flowers (Fig. 4), suggesting that the JcTM6 promoter is a flower-specific promoter. AtIPT4 is a cytokinin biosynthesis gene encoding ATP/ADP isopentenyltransferase. The expression of this gene under the control of $A P 1$ promoter results in the alterations in flower number and organs ( $\underline{\mathrm{Li}}$ et al. 2010). However, the AtIPT4 driven by JcTM6 promoter only gave rise to the changes in flower organs (Fig. 6), indicating that JCTM6 promoter is active at the late stage of flower development rather than floral meristem. This activity is consistent with the expression pattern of the JcTM6 gene in Jatropha. Recently, Ming et al. (2020) showed that JcTM6 promoter has a high activity in female flowers of Jatropha, suggesting that JcTM6 promoter can drive flowerspecific expression of transgenes in different plant species.

When the 842-bp fragment of the JcTM6 promoter ( $-1,717$ to $-876 \mathrm{bp}$ ) was deleted, the promoter was not only active in flowers but also in young leaves (Fig. 5B). We found that the deleted region contained one of the two CArG box motifs, which are very important for mediating the regulatory effect of MADS-box transcription factors (Dolan \& Fields 1991; RichardTreisman 1992). In Jatropha, a fragment of the $J c A P 1$ promoter (from $-1,313$ to $-1,057$ bp), which contains a CArG box motif, is required for promoter activity in inflorescence buds (Tao et al. 2016). The Arabidopsis AP3 promoter contains three CArG boxes: CArG1 is essential for $A P 3$ promoter activity at all stages of flowering; CArG2 is critical for $A P 3$ expression in petals, and $\mathrm{CArG} 3$ represents the binding site of a transcription factor that represses the activity of AP3 promoter during early floral stages (Tilly et al. 1998). Therefore, we propose that the CArG box motif in JcTM6 promoter plays an important role in conferring floral-specific activity in transgenic plants.

Among the floral organs, stamens exhibited the highest activity of JcTM6 promoter (Fig. 4F). This expression pattern could be regulated by pollen-specific elements contained in this promoter, including five GTGA and eight AGAAA motifs. The GTGA motif is critical for the 
240 expression of $g 10$ promoter in tobacco pollen because mutation of the GTGA motif reduced $g 10$ 241 promoter activity in pollen (Rogers et al. 2001). The AGAAA motif, which was identified in the 242 tomato late-stage pollen-specific LAT52 promoter, is necessary for promoter activity during 243 pollen maturation (Bate \& Twell 1998). In potato (Solanum tuberosum L.), the GTGA and 244 AGAAA motifs present in the promoter of $S B g L R$, a pollen-specific gene, are critical for high245 level gene expression in pollen (Lang et al. 2008). In the current study, deletion of an 84283-bp 246 fragment of the JcTM6 promoter, containing four GTGA and two AGAAA motifs, abolished 247 promoter activity in stamens (Fig. 5D). We assumed that these motifs are essential for the 248 activity of the JCTM6 promoter in stamens. Given the importance of CArG box motifs, it is 249 possible that the GTGA and AGAAA motifs cooperate with the CArG box to regulate JcTM6 250 promoter activity in stamens. In addition, although the deleted region contained six AGAAA 251 motifs, these motifs do not seem to be required for JcTM6 promoter activity in stamens. 252 Furthermore, the deleted region also contained a 6-bp quantitative element (Q-element), which 253 plays an enhancer-like role (Hamilton et al. 1998). In maize, deletion of the Q-element from the 254 255 pollen-specific ZM13 promoter reduced the promoter activity by 10-fold (Hamilton et al. 2000). Deletion of the Q-element probably also contributed to the loss of JcTM6 promoter activity in stamens in this study (Fig. 5D). In addition, the deletion variant of the JcTM6 promoter exhibited increased activity in sepals and petals (Fig. 5C and D), indicating the presence of potential negative elements in the deleted region, which inhibit promoter activity in sepals and petals. By the deletion analysis of the JcTM6 promoter, we demonstrate the combination of these elements are of great importance to the promoter activity in the flowers, and detailed studies of the functions of these elements will be conducted in the future.

\section{Conclusions}

Floral-specific promoters play crucial roles in genetic modification of flowering characteristics. In this study, a 1.8-kb JcTM6 promoter fragment was isolated from Jatropha and characterized as a flower-specific promoter in transgenic Arabidopsis plants. When the region from $-1,717$ to $876 \mathrm{bp}$ in the JcTM6 promoter was deleted, the promoter lost its flower-specific activity and gained activity in young leaves. Our results suggest that the JcTM6 promoter could be used to drive flower-specific expression of transgenes in plants.

270

271

\section{Acknowledgements}

272 The authors gratefully acknowledge the Central Laboratory of the Xishuangbanna Tropical

273 Botanical Garden for providing the research facilities.

274

275

References

276

277 
278

279

280

281

282

283

284

285

286

287

288

289

290

291

292

293

294

295

296

297

298

299

300

301

302

303

304

305

306

307

308

309

310

311

312

313

314

315

316

317

318

319

320

321

322

323

324

325

326

Ackerman CM, Yu Q, Kim S, Paull RE, Moore PH, and Ming R. 2008. B-class MADS-box genes in trioecious papaya: two paleoAP3 paralogs, CpTM6-1 and CpTM6-2, and a PI ortholog CPPI. Planta 227:741-753.

Bate N, and Twell D. 1998. Functional architecture of a late pollen promote: pollen-specific transcription is developmentally regulated by multiple stage-specific and co-dependent activator elements. Plant Molecular Biology 37:859-869.

Chen MS, Pan BZ, Wang GJ, Ni J, Niu LJ, and Xu ZF. 2014. Analysis of the transcriptional responses in inflorescence buds of Jatropha curcas exposed to cytokinin treatment. BMC Plant Biology 14:318.

Clough SJ, and Bent AF. 1998. Floral dip: a simplified method for Agrobacterium-mediated transformation of Arabidopsis thaliana. Plant Journal 16:735-743.

Coles JP, Phillips AL, Croker SJ, Garci'a-Lepe R, Lewis MJ, and Hedden P. 1999. Modification of gibberellin production and plant development in Arabidopsis by sense and antisense expression of gibberellin 20-oxidase genes. Plant Journal 17:547-556.

D'Agostino IB, Deruère J, Kieber JJ, 2000. Characterization of the response of the Arabidopsis response regulator gene family to cytokinin. Plant Physiol. 124:1706-1717.

Ding LW, Sun QY, Wang ZY, Sun YB, and Xu ZF. 2008. Using silica particles to isolate total RNA from plant tissues recalcitrant to extraction in guanidine thiocyanate. Analytical Biochemistry 374:426428.

Dolan JW, and Fields S. 1991. Cell-type-specific transcription in yeast. Biochimica et Biophysica Acta 1088:155-169.

Gardner N, Felsheim R, and Smith AG. 2009. Production of male- and female-sterile plants through reproductive tissue ablation. Journal of Plant Physiology 166:871-881. 10.1016/j.jplph.2008.10.002

Hamilton DA, Schwarz YH, Rueda J, Mascarenhas JP. 2000. Comparison of transient and stable expression by a pollen-specific promoter the transformation results do not always agree. Sex Plant Reprod 12:292-295

Hamilton DA, Schwarz YH, and Mascarenhas JP. 1998. A monocot pollen-specific promoter contains separable pollen-specific and quantitative elements. Plant Molecular Biology 38:663-669.

Higo K, Ugawa Y, Iwamoto M, and Korenaga T. 1999. Plant cis-acting regulatory DNA elements (PLACE) database: 1999. Nucleic Acids Research 27:297-300.

Irish VF, and Yamamoto YT. 1995. Conservation of Floral Homeotic Gene-Function between Arabidopsis and Antirrhinum. Plant Cell 7:1635-1644.

Jefferson RA, Kavanagh TA, and Bevan MW. 1987. GUS fusions: beta-glucuronidase as a sensitive and versatile gene fusion marker in higher plants. EMBO Journal 6:3901-3907.

Jeon H-W, Cho J-S, Park E-J, Han K-H, Choi Y-I, and Ko J-H. 2016. Developing xylem-preferential expression of PdGA20oxl, a gibberellin 20-oxidase 1 from Pinus densiflora, improves woody biomass production in a hybrid poplar. Plant Biotechnology Journal 14:1161-1170. 10.1111/pbi.12484

Kumar A, and Sharma S. 2008. An evaluation of multipurpose oil seed crop for industrial uses (Jatropha curcas L.): A review. Industrial Crops and Products 28:1-10.

Lang ZH, Zhou P, Yu JJ, Ao GM, and Zhao Q. 2008. Functional characterization of the pollen-specific SBgLR promoter from potato (Solanum tuberosum L.). Planta 227:387-396.

Lester DR, Ross JJ, Smith JJ, Elliott RC, and Reid JB. 1999. Gibberellin 2-oxidation and the SLN gene of Pisum sativum. Plant Journal 19:65-73.

Li XG, Su YH, Zhao XY, Li W, Gao XQ, and Zhang XS. 2010. Cytokinin overproduction-caused alteration of flower development is partially mediated by $C U C 2$ and $C U C 3$ in Arabidopsis. Gene 450:109-120. 10.1016/j.gene.2009.11.003

Martin DN, Proebsting WM, and Hedden P. 1999. The SLENDER Gene of Pea Encodes a Gibberellin 2-

Peer) reviewing PDF | (2020:01:44947:2:0:NEW 16 Jul 2020) 
327

328

329

330

331

332

333

334

335

336

337

338

339

340

341

342

343

344

345

346

347

348

349

350

351

352

353

354

355

356

357

358

359

360

361

362

363

364

365

366

367

368

369

370

371

372

373

374

375

376

Oxidase. Plant Physiology 121:775-781.

Martino GD, Pan I, Emmanuel E, Levy A, and Irish VF. 2006. Functional analyses of two tomato APETALA3 genes demonstrate diversification in their roles in regulating floral development. Plant Cell 18:1833-1845. 10.1105/tpc.106.042978

Ming X, Tao YB, Fu Q, Tang M, He H, Chen MS, Pan BZ, Xu ZF. 2020. Flower-Specific Overproduction of Cytokinins Altered Flower Development and Sex Expression in the Perennial Woody Plant Jatropha curcas L. Int J Mol Sci 21:640. doi:10.3390/ijms21020640

Muschietti J, Dircks L, Vancanneyt G, and Mccormick S. 1994. LAT52 protein is essential for tomato pollen development: pollen expressing antisense LAT52 RNA hydrates and germinates abnormally and cannot achieve fertilization. Plant Journal 6:321-338.

Muthusamy SK, Sivalingam PN, Sridhar J, Singh D, Haldhar SM, and Kaushal P. 2017. Biotic stress inducible promoters in crop plants- a review. Journal of Agriculture and Ecology 4:14-24.

Nishimura C, Ohashi Y, Sato S, Kato T, Tabata S, Ueguchi C, 2004. Histidine kinase homologs that act as cytokinin receptors possess overlapping functions in the regulation of shoot and root growth in Arabidopsis. Plant Cell 16: 1365-1377.

Pan BZ, and Xu ZF. 2011. Benzyladenine Treatment Significantly Increases the Seed Yield of the Biofuel Plant Jatropha curcas. Journal of Plant Growth Regulation 30:166-174.

Pnueli L, Abu-abeid M, Zamir D, Nacken W, Schwarz-Sommer Z, and Lifschitz E. 1991. The MADS box gene family in tomato: temporal expression during floral development, conserved secondary structures and homology with homeotic genes from Antirrhinum and Arabidopsis. Plant Journal 1:255-266.

Potenza C, Aleman L, and Sengupta-Gopalan C. 2004. Targeting transgene expression in research, agricultural, and environmental applications: Promoters used in plant transformation. In Vitro Cellular \& Developmental Biology - Plant 40:1-22. 10.1079/IVP2003477

Raju AJS, and Ezradanam V. 2002. Pollination ecology and fruiting behaviour in a monoecious species, Jatropha curcas L. (Euphorbiaceae). Current Science 83:1395-1398.

Rao GR, Korwar GR, Shanker AK, and Ramakrishna YS. 2008. Genetic associations, variability and diversity in seed characters, growth, reproductive phenology and yield in Jatropha curcas (L.) accessions. Trees-Structure and Function 22:697-709. 10.1007/s00468-008-0229-4

Rijpkema AS, Royaert S, Zethof J, van der Weerden G, Gerats T, and Vandenbussche M. 2006. Analysis of the Petunia TM6 MADS box gene reveals functional divergence within the DEF/AP3 lineage. Plant Cell 18:1819-1832.

Rogers HJ, Bate N, Combe J, Sullivan J, Sweetman J, Swan C, Lonsdale DM, and Twell D. 2001. Functional analysis of cis-regulatory elements within the promoter of the tobacco late pollen gene g10. Plant Molecular Biology 45:577-585.

Roque E, Gómez MD, Ellul P, Wallbraun M, Madueño F, Beltrán JP, and Cañas LA. 2007. The PsEND1 promoter: a novel tool to produce genetically engineered male-sterile plants by early anther ablation. Plant Cell Reports 26:313-325. 10.1007/s00299-006-0237-z

Sakamoto T, Morinaka Y, Ishiyama K, Kobayashi M, Itoh H, Kayano T, Iwahori S, Matsuoka M, and Tanaka H. 2003. Genetic manipulation of gibberellin metabolism in transgenic rice. Nature Biotechnology 21:909-913. 10.1038/nbt847

Siebert PD, Chenchik A, Kellogg DE, Lukyanov KA, and Lukyanov SA. 1995. An improved PCR method for walking in uncloned genomic DNA. Nucleic Acids Research 23:1087-1088.

Tao YB, He LL, Niu LJ, and Xu ZF. 2015. Isolation and Characterization of an Ubiquitin Extension Protein Gene (JcUEP) Promoter From Jatropha Curcas. Planta 241: 826-836.

Tao YB, He LL, Niu LJ, and Xu ZF. 2016. Isolation and characterization of the Jatropha curcas APETALA1 (JCAPl) promoter conferring preferential expression in inflorescence buds. Planta 244:467-478.

Tewari JP, Dwivedi HD, Pathak M, Srivastava SK. 2007. Incidence of a mosaic disease in Jatropha curcas L. from eastern Uttar Pradesh. Curr Sci 93:1048-1049.

Peer] reviewing PDF | (2020:01:44947:2:0:NEW 16 Jul 2020) 
377 378 379 380 381 382 383 384 385 386 387 388 389 390 391 392 393
Thomas SG, Phillips AL, and Hedden P. 1999. Molecular cloning and functional expression of gibberellin 2- oxidases, multifunctional enzymes involved in gibberellin deactivation. Proceedings of the National Academy of Sciences of the United States of America 96:4698-4703. Tilly JJ, Allen DW, and Jack T. 1998. The CArG boxes in the promoter of the Arabidopsis floral organ identity gene APETALA3 mediate diverse regulatory effects. Development 125:1647-1657.

Treisman R. 1992. The serum response element. Trends in Biochemical Sciences 17:423-426.

Verdonk JC, Shibuya K, Loucas HM, Colquhoun TA, Underwood BA, and Clark DG. 2008. Flowerspecific expression of the Agrobacterium tumefaciens isopentenyltransferase gene results in radial expansion of floral organs in Petunia hybrida. Plant Biotechnology Journal 6:694-701. 10.1111/j.1467-7652.2008.00349.x

Weigel D, and Meyerowitz EM. 1994. The ABCs of Floral Homeotic Genes. Cell 78:203-209.

Wu M, Fan SL, Song MZ, Pang CY, Wei JH, Liu J, Yu JW, Zhang JF, and Yu SX. 2011. Cloning and expression of GhTM6, a gene that encodes a B-class MADS-box protein in Gossypium hirsutum. Russian Journal of Plant Physiology 58:498-506.

Zhang L, He LL, Fu QT, and Xu ZF. 2013. Selection of Reliable Reference Genes for Gene Expression Studies in the Biofuel Plant Jatropha curcas Using Real-Time Quantitative PCR. International Journal of Molecular Sciences 14:24338-24354. 


\section{Table 1 (on next page)}

Sequences of the primers used in this study 
1 Table 1. Sequences of the primers used in this study

2

\begin{tabular}{|c|c|c|}
\hline Name & Sequence ( $5^{\prime}$ to $\left.3^{\prime}\right)$ & Feature \\
\hline GSP1 & CTCTTGGAATAAGTAACCTGTCTGTTGG & $\begin{array}{l}J c T M 6 \text { gene-specific primer for } \\
\text { genome walking }\end{array}$ \\
\hline GSP2 & CAAAACCCACTACTACAAAACCGAAGA & $\begin{array}{l}J_{c T M 6} \text { gene-specific primer for } \\
\text { genome walking }\end{array}$ \\
\hline XT95 & GCTGCTAAGGCTGTTGGGAA & $\begin{array}{l}J_{c} G A P D H \text { gene primer for } \mathrm{qRT}- \\
\text { PCR }\end{array}$ \\
\hline XT96 & GACATAGCCCAATATTCCCTTCAG & $\begin{array}{l}J_{c} G A P D H \text { gene primer for } \mathrm{qRT}- \\
\text { PCR }\end{array}$ \\
\hline XK712 & TATCTCTTCGGTTTTGTAGTAGTGGG & JcTM6 gene primer for qRT-PCR \\
\hline XK713 & TCTCTTGGAATAAGTAACCTGTCTGT & $J c T M 6$ gene primer for $\mathrm{qRT}-\mathrm{PCR}$ \\
\hline XT405 & TGCTCTAGAAATAGCTATAAAATCAATT & $\begin{array}{l}\text { For cloning the full-length } \\
\text { promoter and construction of } \\
\text { JCTM6:GUS }\end{array}$ \\
\hline XT408 & CGCGGATCCTTTTCCTTTCTTCTTGATA & $\begin{array}{l}\text { For cloning the full-length } \\
\text { promoter and construction of } \\
\text { JcTM6:GUS }\end{array}$ \\
\hline XD548 & GCTCTAGACGCTTACAGAATTTGCGA & For construction of $D: G U S$ \\
\hline XB994 & CAATCTTTCCACGACCCATTTTTCCTT & $\begin{array}{l}\text { JcTM6 gene-specific primer for } 5 \text { '- } \\
\text { RACE }\end{array}$ \\
\hline XK718 & TGTGCCAATCTACGAGGGTTT & Atactin gene primer for $\mathrm{qRT}-\mathrm{PCR}$ \\
\hline XK719 & TTTCCCGCTCTGCTGTTGT & Atactin gene primer for $\mathrm{qRT}-\mathrm{PCR}$ \\
\hline XK984 & TCGCTGAGTTCCACCGCTCTAAG & AtIPT4 gene primer for $\mathrm{qRT}-\mathrm{PCR}$ \\
\hline XK985 & AGGGTCCCATTTATCCATGTCATTG & AtIPT4 gene primer for $\mathrm{qRT}-\mathrm{PCR}$ \\
\hline XE815 & CCTTGTCAATGGCAAGAAGAGGCAA & $\begin{array}{l}\text { AHK2 gene primer for qRT-PCR } \\
\text { (Nishimura et al, 2004) }\end{array}$ \\
\hline XE816 & CACCTTCTGCAACTCGTCTGTT & $A H K 2$ gene primer for qRT-PCR \\
\hline XE819 & TCAGAGAACATCTTGCCTCGT & $A R R 5$ gene primer for qRT-PCR \\
\hline XE820 & AGCTGCGAGTAGATATCATTAGCTT & ARR5 gene primer for $\mathrm{qRT}-\mathrm{PCR}$ \\
\hline
\end{tabular}




\section{Figure 1}

A comparison of JCTM6 and its homologs.

(A) The alignment of the deduced amino acid sequences of JcTM6 with that of Vitis vinifera VvTM6 (accession No.DQ979341), Carica papaya CpTM6-1 (accession No.ABQ51321), and CpTM6-2 (accession No.ABQ51322), Populus trichocarpa PTD (accession No.AAC13695) , Gossypium hirsutum GhTM6 (accession No.ADX60056), Petunia x hybrida PhTM6 (accession No.AF230704) and Solanum lycopersicum SITM6 (accession No.CAA43171). Identically and partially conserved amino acid sequences are shown in black and gray, respectively. The conserved regions, MADS domain and $\mathrm{K}$ domain and paleoAP3 C-terminal motif in JCTM6 are underlined. (B) A phylogenetic analysis of JCTM6 and other homologs. Jatropha curcas JCDEF (accession No. XP_012071964), Solanum lycopersicum TAP3 ( accession No. ABG73412), Vitis vinifera VvAP3 (accession No. NP_001267960), Arabidopsis thaliana AP3 (accession No. BAA04665), Petunia hybrida PMADS1 (accession No. Q07472). The tree was constructed using MEGA 7.0 software and the neighbor-joining ( $\mathrm{N}-\mathrm{J})$ method. The $\mathrm{N}-\mathrm{J}$ unrooted dendrogram was generated from an alignment of the deduced amino acids with the ClustalW program. One thousand replicates were used for the Bootstrap test. The scale bar indicates the average number of substitutions per site. 
A

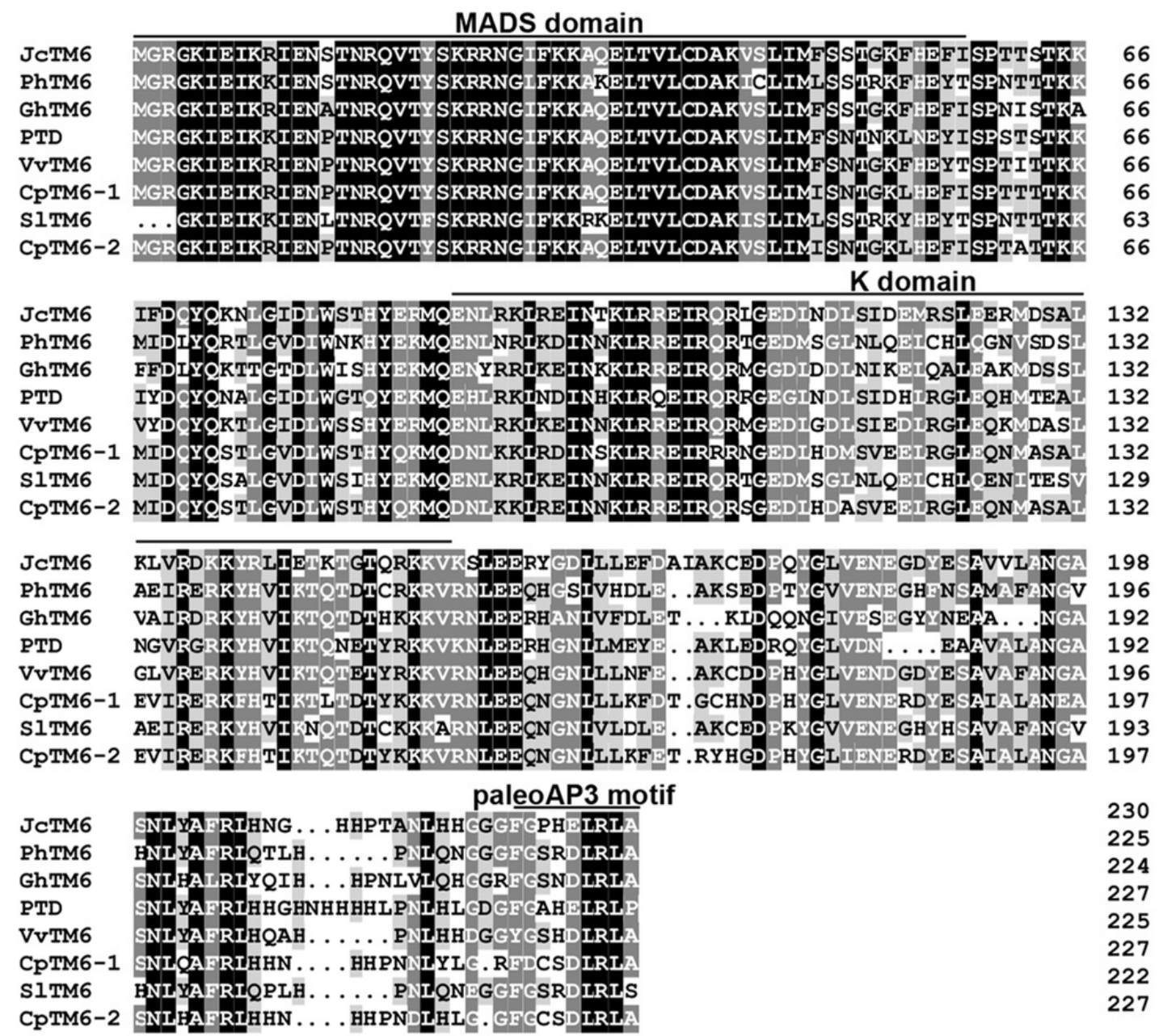

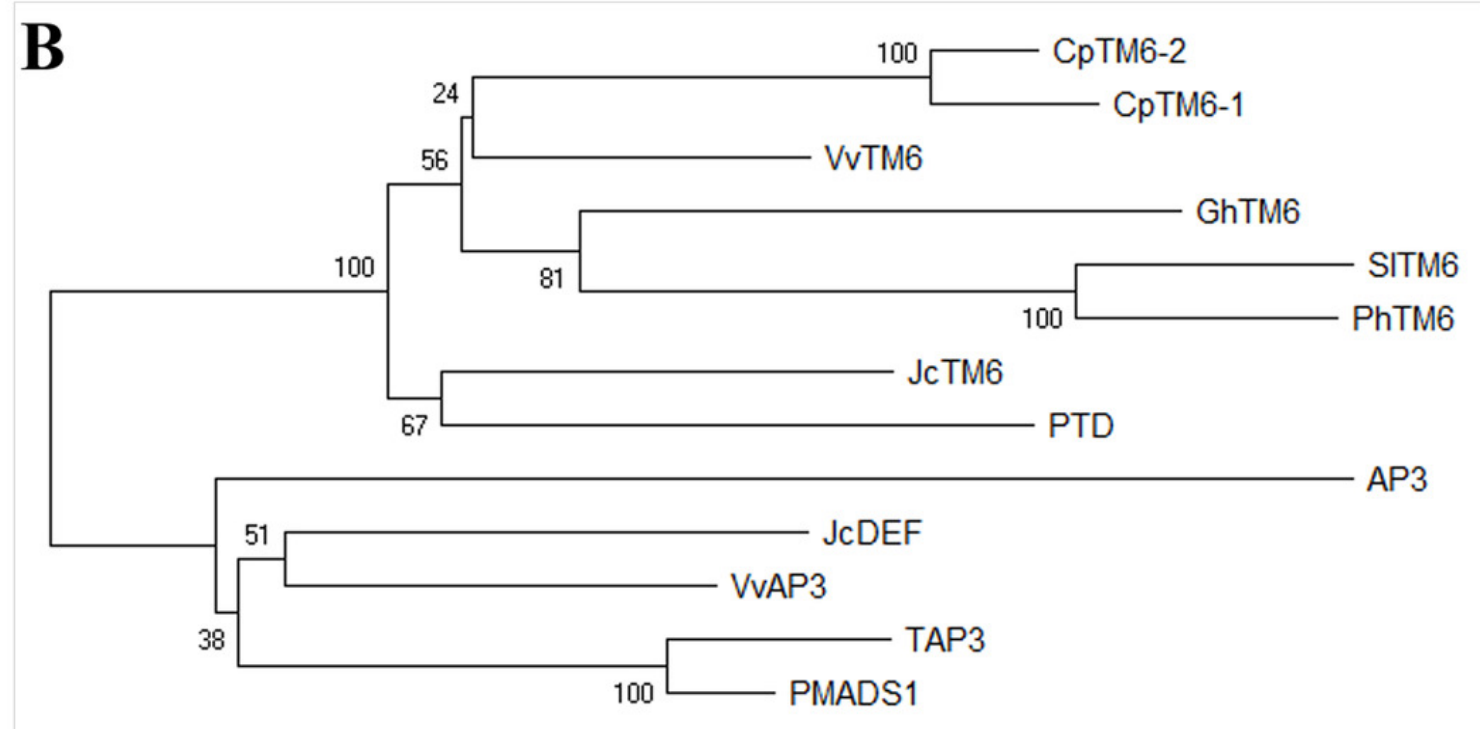

0.10 


\section{Figure 2}

Expression pattern of JCTM6 in Jatropha.

Samples from adult plants: roots $(R)$, stems $(S)$, young leaves $(\mathrm{YL})$, mature leaves $(\mathrm{ML})$, inflorescence buds (If), female flowers (FF), male flowers (MF), pericarps at 42 days after pollination (DAP) (Pp 42d), seeds at 42 DAP (Sd 42d), male sepals (MS), male petals (MP), stamens (St), female sepals (FS), female petals (FP), and pistils (Pi). qRT-PCR results were obtained from three biological replicates. The errors denote the SD. The values were normalized to the expression of JCGAPDH (Zhang et al. 2013). The relative expression level of young leaves was set as the standard value of 1 .

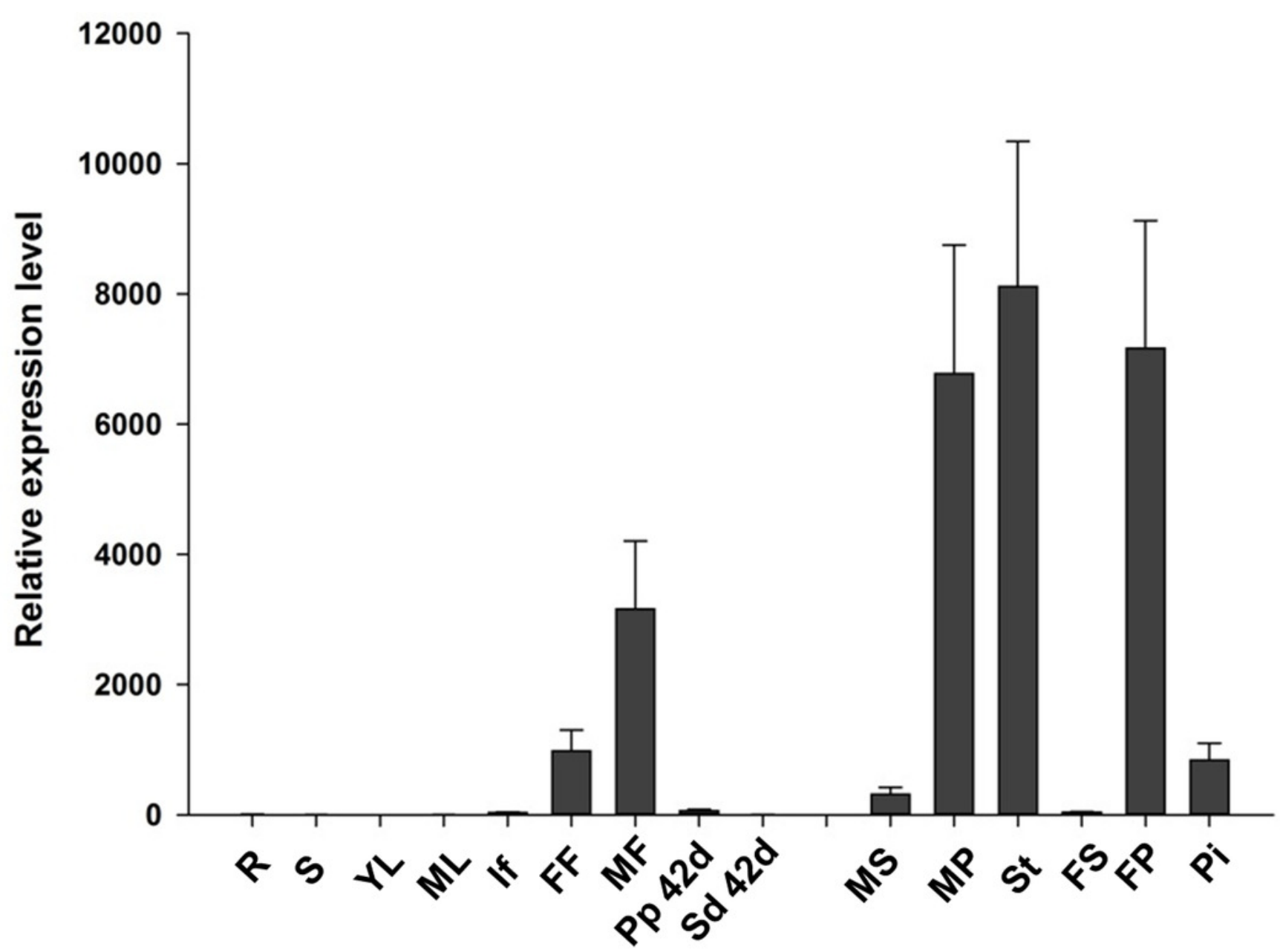


Figure 3

JCTM6 promoter sequence and promoter-reporter gene construct.

(A) The nucleotide sequence of the JcTM6 promoter. The transcription start site $(+1)$ is in red. The start codon ATG is in bold and boxed. Putative regulatory elements on both strands are shown in bold and underlined. (B) A schematic of the T-DNA regions of the JcTM6:GUS binary vector used for transformation. 


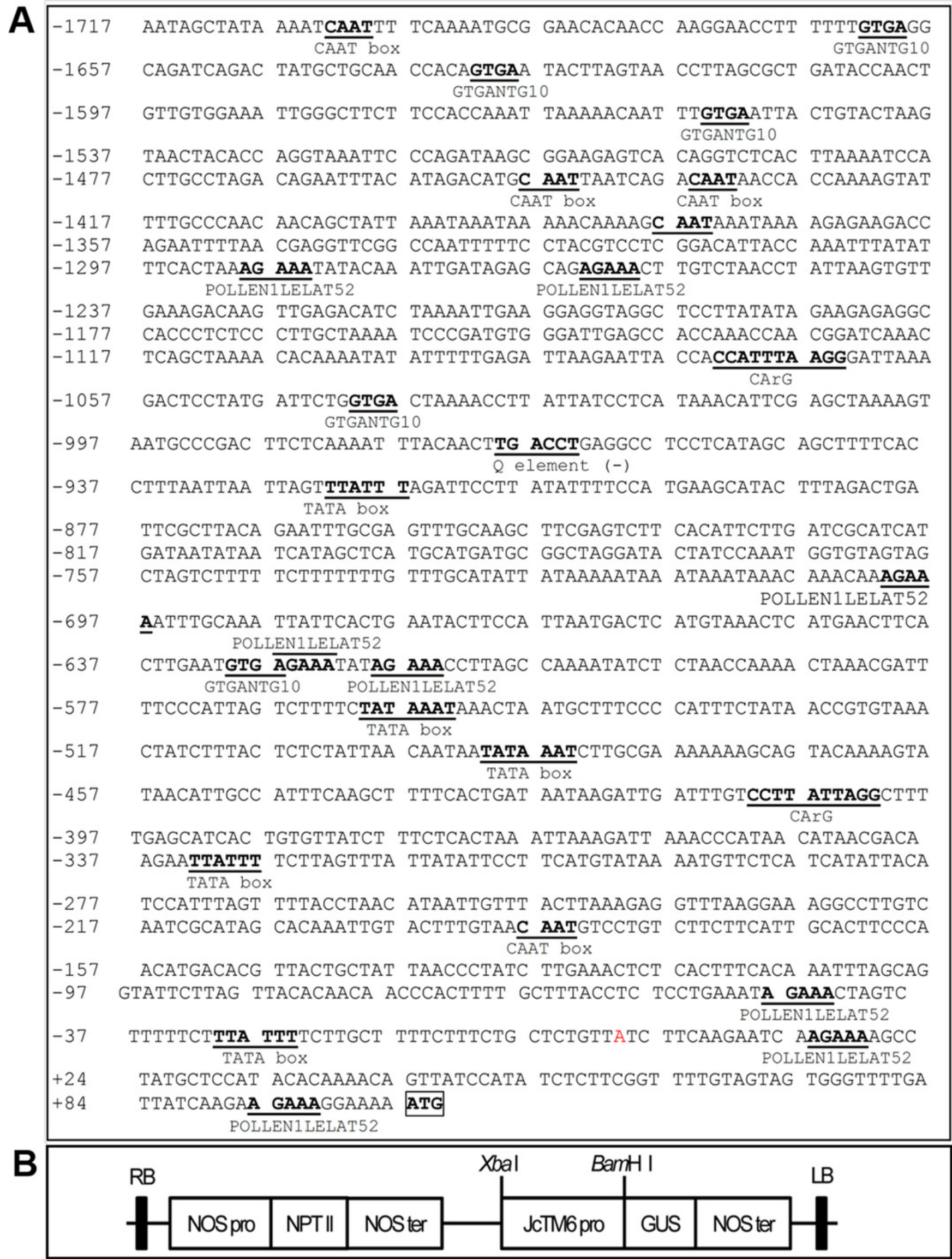

Peer) reviewing PDF | (2020:01:44947:2:0:NEW 16 Jul 2020) 
Figure 4

Histochemical GUS staining of transgenic Arabidopsis harboring the JCTM6:GUS fusion.

(A) Ten-day-old seedlings, (B) roots, (C) stems, (D) leaves, (E) inflorescence buds, (F) open

flowers, (G) green siliques. Pe, petals; Se, sepals; St, stamens. Red bars $=1 \mathrm{~mm}$, black bars $=2 \mathrm{~mm}$. 


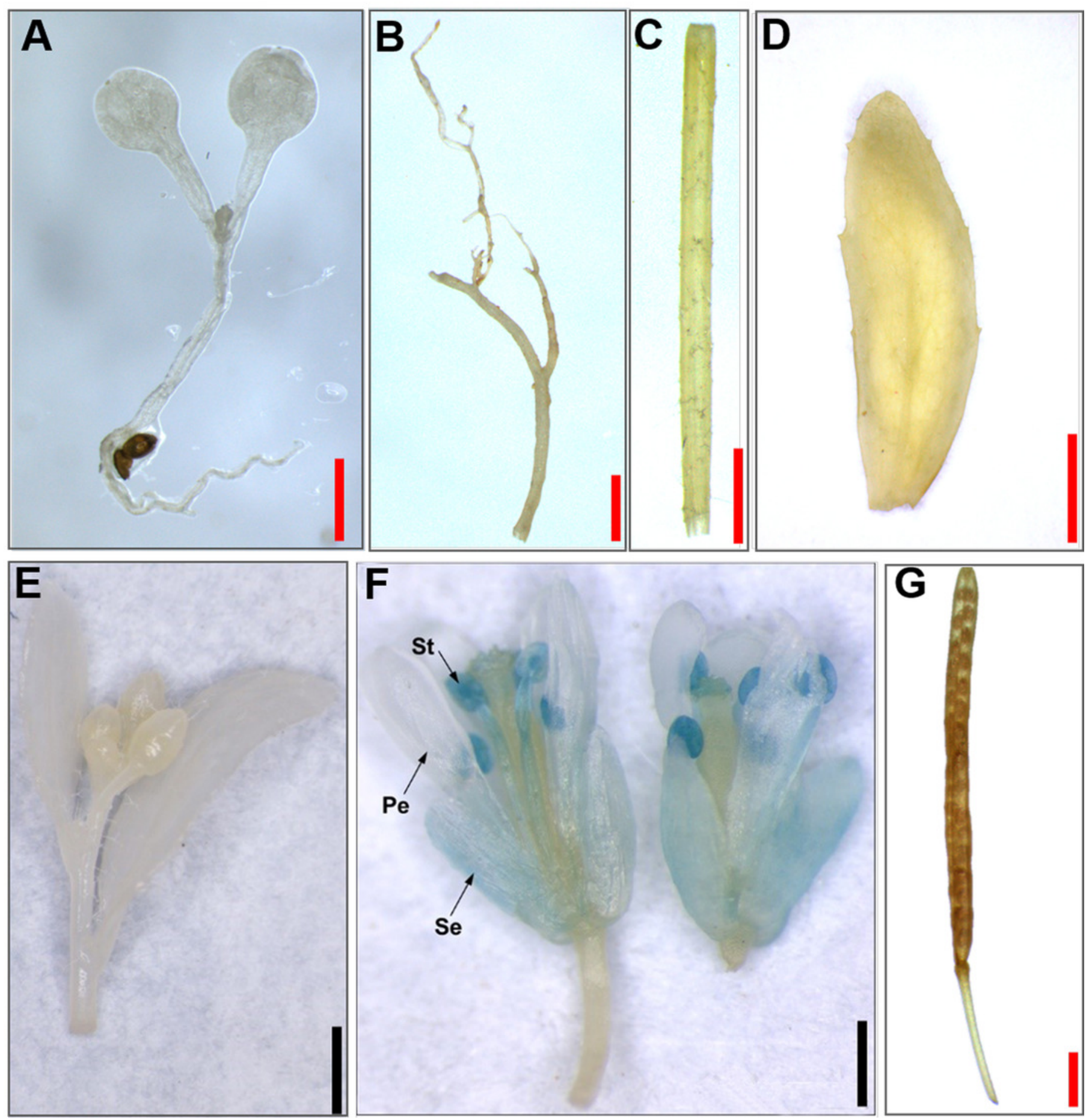




\section{Figure 5}

Histochemical GUS staining of transgenic Arabidopsis harboring the JcTM6 deletion.

(A) Schematic representation of JCTM6 promoter deletion. FL, full length JcTM6 promoter, De'l, deletion. GTGA: GTGANTG10 motif (gray vertical bars), AGAAA: POLLEN1LELAT52 motif (black vertical bars), CArG box: CWWWWWWWWG (yellow vertical bars), Q-element: TGACCT (red vertical bar). (B) young leaves, (C) flower buds, (D) flowers. Pe, petals; Se, sepals; St, stamens. Red bar $=1 \mathrm{~mm}$, black bars $=0.5 \mathrm{~mm}$.

A
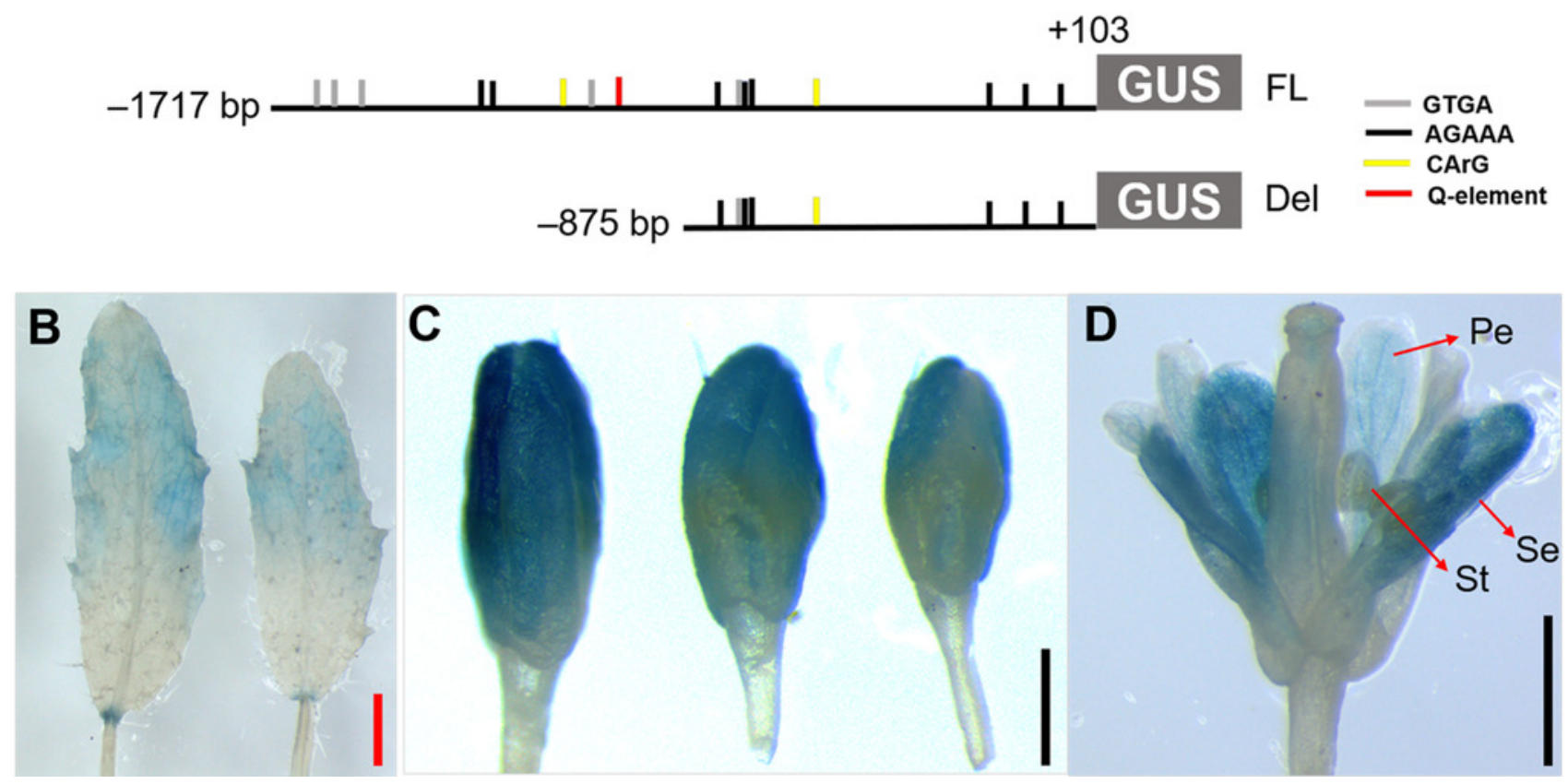
Figure 6

Flower size is increased in transgenic JCTM6:AtIPT4 Arabidopsis.

Inflorescences of wild-type (A) and transgenic L1 (B) and L22 (C) lines. Flowers of wild-type and transgenic L1 and L22 lines (D). Dissected flowers of WT and transgenic L1 and L22 lines (E). Se, sepals; Pe, petals; St, stamens; Ca, carpels; WT, wild-type. White bars $=3 \mathrm{~mm}$, yellow $\mathrm{bar}=2 \mathrm{~mm}$. 

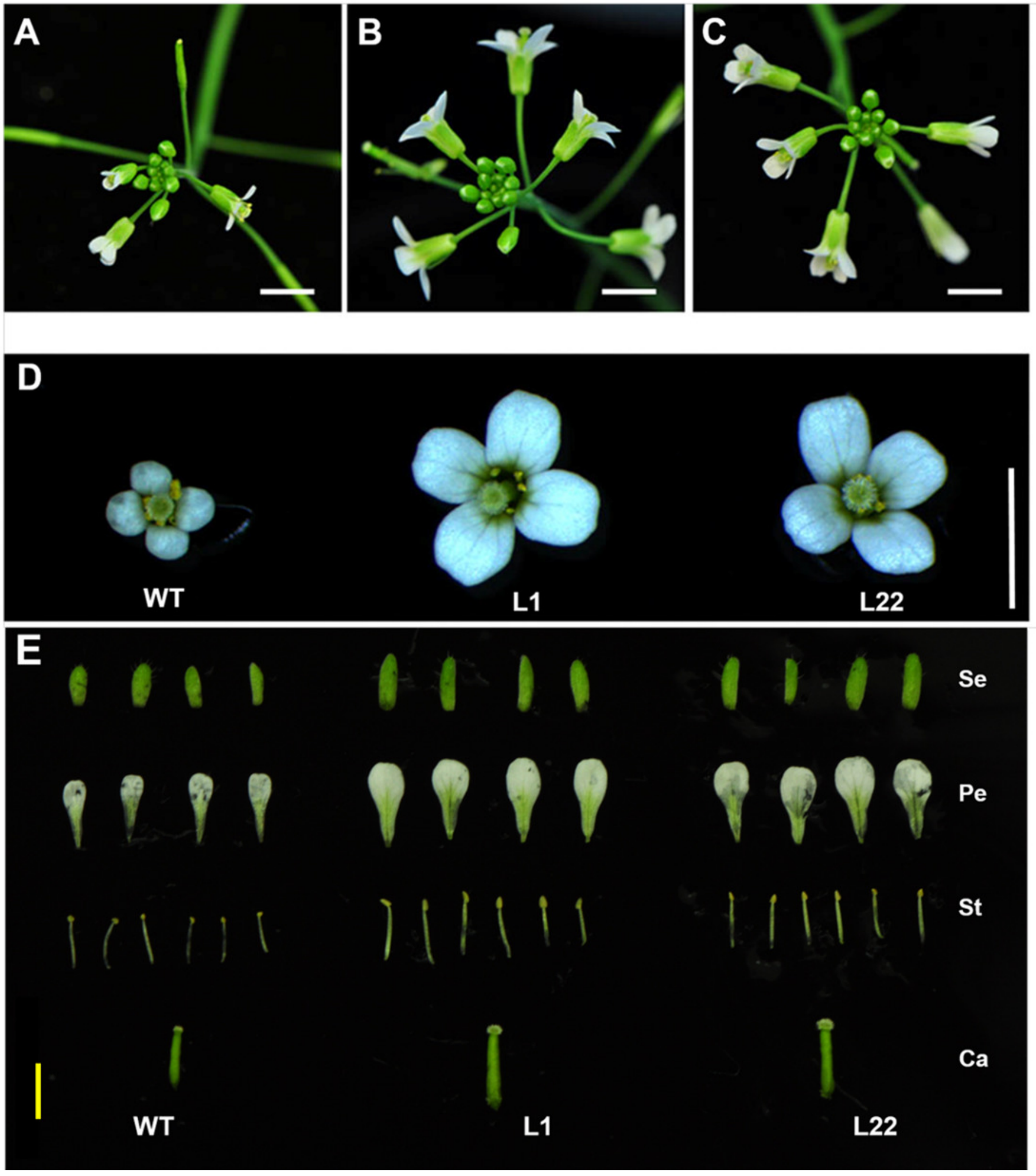


\section{Figure 7}

The expression analysis of AtIPT4, AHK2 and ARR5 in JCTM6:AtIPT4 transgenic Arabidopsis.

(A) The expression levels of AtIPT4 in the leaves and flowers of wild type (WT) plants and transgenic lines (L1 and L22). (B) The expression levels of AHK2 and ARR5 in the flowers of wild type (WT) plants and transgenic lines (L1 and L22). The values represent the means \pm standard deviation $(n=3)$. Student's t-test was used to determine significant differences. * $p$ $\leq 0.05, * * p \leq 0.01$. 

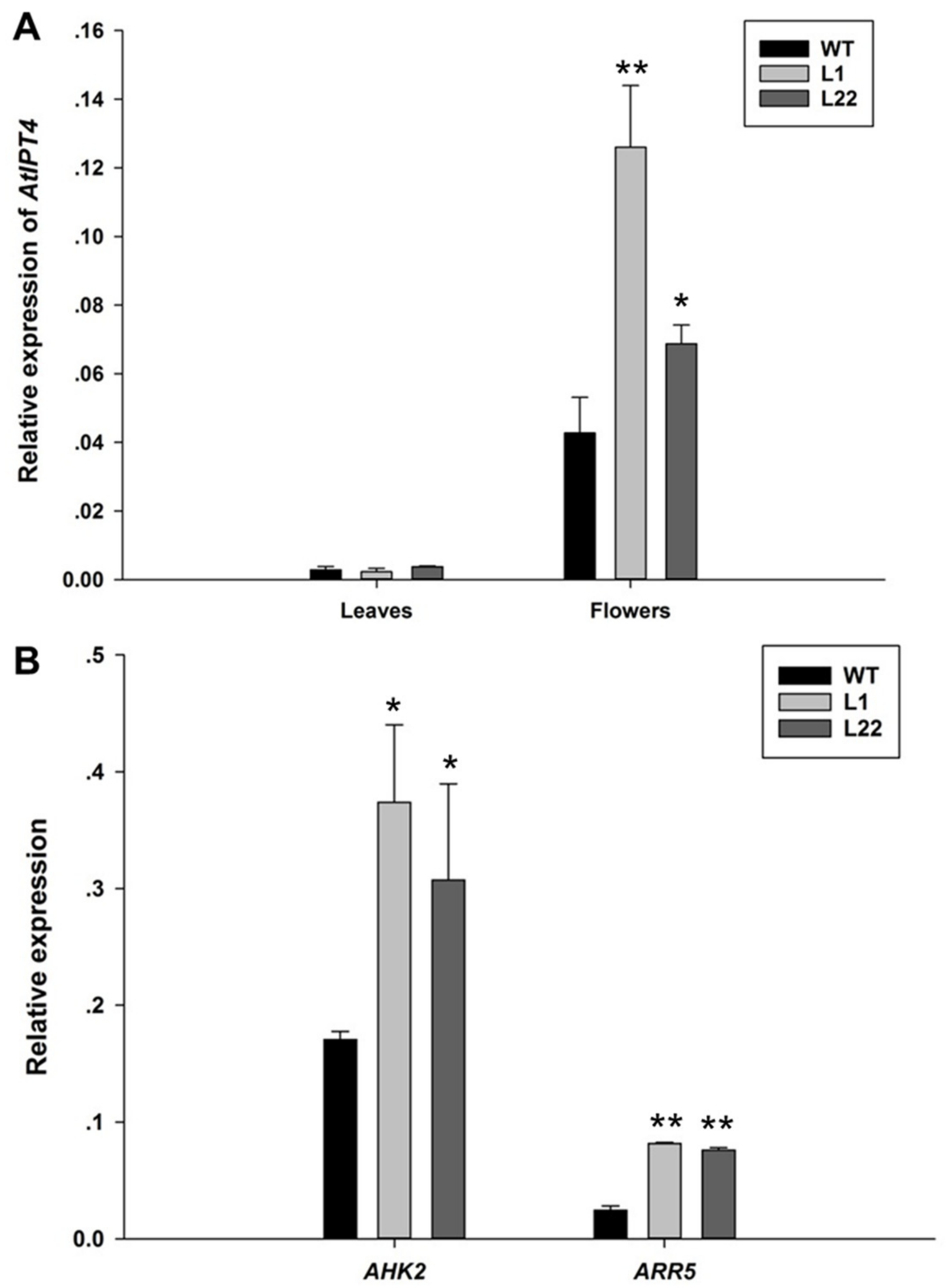